\title{
Fast Machine Learning Annotation in the Medical Domain: A Semi-Automated Video Annotation Tool for Gastroenterologists
}

\section{Adrian Krenzer ( $\nabla$ adrian.krenzer@uni-wuerzburg.de )}

Julius-Maximilians-Universitat Wurzburg https://orcid.org/0000-0002-1593-3300

\section{Kevin Makowski}

University of Würzburg: Julius-Maximilians-Universitat Wurzburg

\section{Amar Hekalo}

University of Würzburg: Julius-Maximilians-Universitat Wurzburg

Daniel Fitting

Universitätsklinikum Würzburg: Universitatsklinikum Wurzburg

Joel Troya

University Hospital Wurzburg: Universitatsklinikum Wurzburg

\section{Wolfram G. Zoller}

City of Stuttgart Hospitals Katharinenhospital: Klinikum Stuttgart Katharinenhospital

\section{Alexander Hann}

Universitätsklinikum Würzburg: Universitatsklinikum Wurzburg

\section{Frank Puppe}

Universität Würzburg: Julius-Maximilians-Universitat Wurzburg

\section{Research}

Keywords: Machine learning, Deep learning, Annotation, Endoscopy, Gastroenterology, Automation, Object detection

Posted Date: August 10th, 2021

DOI: https://doi.org/10.21203/rs.3.rs-776478/v1

License: (c) (i) This work is licensed under a Creative Commons Attribution 4.0 International License. Read Full License 
Correspondence:

adrian.krenzer@uni-wuerzburg.de

${ }^{1}$ Department of Artificial

Intelligence and Knowledge

Systems, Sanderring 2, 97070

Würzburg, Germany

Full list of author information is available at the end of the article

\begin{abstract}
Background: Machine learning, especially deep learning, is becoming more and more relevant in research and development in the medical domain. For all of the supervised deep learning applications, data is the most critical factor in securing successful implementation and sustaining the progress of the machine learning model. Especially gastroenterological data, which often involves endoscopic videos, are cumbersome to annotate. Domain experts are needed to interpret and annotate the videos. To support those domain experts, we generated a framework. With this framework, instead of annotating every frame in the video sequence, experts are just performing key annotations at the beginning and the end of sequences with pathologies, e.g. visible polyps. Subsequently, non-expert annotators supported by machine learning add the missing annotations for the frames in-between.
\end{abstract}

Results: Using this framework we were able to reduce work load of domain experts on average by a factor of 20 . This is primarily due to the structure of the framework, which is designed to minimize the workload of the domain expert. Pairing this framework with a state-of-the-art semi-automated pre-annotation model enhances the annotation speed further. Through a study with 10 participants we show that semi-automated annotation using our tool doubles the annotation speed of non-expert annotators compared to a well-known state-of-the-art annotation tool.

Conclusion: In summary, we introduce a framework for fast expert annotation for gastroenterologists, which reduces the workload of the domain expert considerably while maintaining a very high annotation quality. The framework incorporates a semi-automated annotation system utilizing trained object detection models. The software and framework are open-source.

Keywords: Machine learning; Deep learning; Annotation; Endoscopy; Gastroenterology; Automation; Object detection

\section{Background}

Machine learning especially deep learning is becoming more and more relevant in research and development in the medical domain [1,2]. For all of the supervised deep learning applications, data is the most critical factor in securing successful implementation and sustaining progress. Numerous studies have shown that access to data and data quality are crucial to enable successful machine learning of medical diagnosis, providing real assistance to physicians $[3,4,5,6,7]$. Exceptionally 
high-quality annotated data can improve deep learning detection results to great extent $[8,9,10]$. E.g., Webb et al. show that higher data quality improves detection results more than using larger amounts of lower quality data [11]. This is especially important to keep in mind while operating in the medical domain, as mistakes may have fatal consequences. Nevertheless, acquiring such data is very costly particularly if domain experts are involved. On the one hand domain, experts have minimal time resources for data annotation, while on the other hand, data annotation is a highly time-consuming process. The best way to tackle this problem is by reducing the annotation time spend by the actual domain expert as much as possible while using non-experts to finish the process. Therefore, in this paper, we designed a framework that utilizes a two-step process involving a small expert annotation part and a large non-expert annotation part. This shifts most of the workload from the expert to a non-expert while still maintaining proficient high-quality data. Both of the tasks are combined with AI to enhance the annotation process efficiency further. To handle the entirety of this annotation process, we introduce the software Fast Colonoscopy Annotation Tool (FastCat). This tool assists in the annotation process in endoscopic videos but can easily be extended to any other medical domain. The main contributions of our paper are:

1) We introduce a framework for fast expert annotation, which reduces the workload of the domain expert by a factor of 20 while maintaining very high annotation quality.

2) We publish an open-source software for annotation in the gastroenterological domain and beyond, including two views, one for expert annotation and one for non-expert annotation. ${ }^{[1]}$

3) We incorporate a semi-automated annotation process in the software, which reduces the annotation time of the annotators and further enhances the annotation process's quality.

To overview existing work and properly allocate our paper in the literature we describe a brief history reaching from general annotation tools for images and videos to annotation specialized for medical use.

\section{A brief history of annotation tools}

As early as the 1990s, the first methods were conceived to collect large datasets of labeled images [12]. E.g., "The Open Mind Initiative", a web-based framework, was developed in 1999. Its goal was to collect annotated data by web users to be utilized by intelligent algorithms [13]. Over the years, various ways to obtain annotated data have been developed. E.g., an online game called ESP was developed to generate labeled images. Here, two random online players are given the same image and, without communication, must guess the thoughts of the other player about the image and provide a common term for the target image as quickly as possible $[14,12]$. As a result, several million images have been collected. The first and foremost classic annotation tool called labelme was developed in 2007 and is still one of the most popular open-source online annotation tools to create datasets

${ }^{[1]}$ https://github.com/fastcatai/fastcat 
Table 1 Comparison between video and image annotation tools.

\begin{tabular}{|c|c|c|c|c|c|c|}
\hline \multicolumn{2}{|c|}{ Tool } & CVAT & Labellmg & labelme & VoTT & VIA \\
\hline & Image & $\bullet$ & $\bullet$ & $\bullet$ & $\bullet$ & $\bullet$ \\
\hline & Video & - & - & - & - & - \\
\hline & Usability & Easy & Easy & Medium & Medium & Hard \\
\hline \multirow{5}{*}{$\begin{array}{l}\frac{n}{\pi} \\
\frac{n}{ \pm} \\
\dot{0} \\
亡\end{array}$} & VOC & $\bullet$ & $\bullet$ & $\bullet$ & $\bullet$ & - \\
\hline & $\mathrm{COCO}$ & $\bullet$ & - & $\bullet$ & - & $\bullet$ \\
\hline & YOLO & $\bullet$ & $\bullet$ & - & - & - \\
\hline & TFRecord & $\bullet$ & - & - & $\bullet$ & - \\
\hline & Others & - & - & $\bullet$ & $\bullet$ & $\bullet$ \\
\hline
\end{tabular}

for computer vision. Labelme provides the ability to label objects in an image by specific shapes, as well as other features [15]. From 2012 to today, with the rise of deep learning in computer vision, the number of annotation tools expanded rapidly. One of the most known and contributing annotation tools is LabelImg, published in 2015. LabelImg is an image annotation tool based on Python which utilizes bounding boxes to annotate images. The annotations are stored in XML files that are saved in either PASCAL VOC or YOLO format. Additionally, in 2015 Playment was introduced. Playment is an annotation platform to create training datasets for computer vision. It offers labeling for images and videos using different $2 \mathrm{D}$ or $3 \mathrm{D}$ boxes, polygons, points, or semantic segmentation. Besides, automatic labeling is provided for support. In 2017 Rectlabel entered the field. RectLabel is a paid labeling tool that is only available on macOS. It allows the usual annotation options like bounding boxes as well as automatic labeling of images. It also supports the PASCAL VOC XML format and exports the annotations to different formats (e.g., YOLO or COCO JSON). Next, Labelbox, a commercial training data platform for machine learning, was introduced. Among other things, it offers an annotation tool for images, videos, texts, or audios and data management of the labeled data. Nowadays, a variety of image and video annotation tools can be found. Some have basic functionalities, and others are designed for particular tasks. We picked five freely available state-of-the-art annotation tools and compared them more in-depth. In table 1 , we shortly describe these tools and compare them.

Computer Vision Annotation Tool (CVAT) CVAT [16] was developed by Intel and is a free and open-source annotation tool for images and videos. It is based on a client-server model, where images and videos are organized as tasks and can be split up between users to enable a collaborative working process. Files can be inserted onto the server through a remote source, mounted file system, or uploading from the local computer. Before a video can be annotated, it must be partitioned into its frames, which then can be annotated. Several annotation formats are supported, including the most common formats such as VOC, COCO, YOLO and TFRecord. Available annotation shapes and types are labeling, bounding boxes, polygons, polylines, dots, and cuboids. CVAT also includes features for a faster annotation process in videos. The disadvantages of this tool are that it currently only supports the Google Chrome browser, and due to the Chrome Sandbox, performance issues could appear.

LabelImg LabelImg [17] is an image annotation tool that is written in Python and uses the Qt library as a graphical user interface. It can load a bulk of images but only 
supports bounding box annotations and saves it as a XML file in VOC or YOLO format. The functionalities are minimal but sufficient for manual annotation of images. Furthermore, it does not contain any automatic or semi-automatic features which could speed up the process.

labelme The annotation tool labelme [18] is written in Python, uses Qt as its graphical interface and only supports image annotation. It is advertised that videos could be annotated with this tool, but no video annotation function was found and the user must manually extract all frames from the video beforehand. Also, there are no automatic or semi-automatic features available and uses basic shapes like polygons, rectangles, circles, points, lines and polylines to annotate images. It uses its annotation data format, but it can be converted into the VOC format for semantic and instance segmentation and the COCO format is only available for instance segmentation.

Visual Object Tagging Tool (VoTT) Microsoft's tool VoTT [19] is open-source and can be used for images and videos. Since it is written in TypeScript and uses the React library as a user interface, it is possible to use it as a web application that can run in any web browser. Alternatively, it can also run locally as a native application with access to the local file system. Images and videos are introduced to the program via a connected entity. This can be a path on the local file system, a Bing image search query via an API key, or secure access to an Azure Blob Storage resource. Available annotation shapes are rectangles and polygons that can be tagged. These can then be exported for the Azure Custom Vision Service and Microsoft Cognitive Toolkit (CNTK). Also, the following formats are available: VOC, TFRecord, CSV and a VoTT-specific JSON. Videos are also extracted into their frames, but one can set a frame extraction rate (FER) to control the frequency in which the frames are extracted, i.e., a FER of 1 only extracts one frame every second and a FER of 10 is saving a frame every tenth of a second. Because a timestamp of the video is stored along with the extracted frame, it should be noted that between frames and the corresponding timestamp can be inaccurate due to rounding errors or a discrepancy between the FER and actual video frame rate. The FER value should be set to the actual frame rate of the video, to get the best possible accuracy, It is also important to mention that VoTT relies on the HTML5 Video element, which has limited video formats and can vary between browsers.

VGG Image Annotator (VIA) VIA [20,21] is a tool that runs in a web browser without further installation and is only build from HTML, JavaScript, and CSS. It can import and export annotations from COCO and a VIA-specific CSV and JSON. The available annotation shapes are polygons, rectangles, ellipses, lines, polylines, and points. Video annotation features the annotation of temporal segments to mark e.g., a particular activity within the video. Defined segments of the track can also annotate an audio file. VIA does not contain any automatic functionalities within the tool itself; these are relatively independent steps. These steps can be broken down to: Model predicts on frames, save predictions so that they can be imported into VIA, and lastly, check and update annotations if necessary. 
Medical annotation tools

With the considerable increase in interest and progress in machine learning in our society the need for machine learning models shifts in different domains including medicine. Thus, artificial intelligence can be used to assist medical professionals in their daily routines $[22,23,24]$. As a result, the need for labeled medical images and videos is also a major issue for medical professionals. While it is possible to use common annotation tools such as those already described above, some annotation tools have already been adapted to medical conditions. A well-known example from 2004 is "ITK-Snap", a software for navigating and segmenting three-dimensional medical image data [25]. Another example is "ePAD", an open-source platform for segmentation of 2D and 3D radiological images [26]. The range of medical segmentation tools has become very broad nowadays, as they are usually specialized for many different areas of medicine. Another annotation tool published in 2015 is TrainingData $[27,28]$. TrainingData is a typical annotation tool for labeling machine learning (computer vision) training images and videos. This product offers good features, including labeling support through built-in machine learning models. TrainingData also supports DICOM (Digital Imaging and Communications in Medicine), a widespread format in the medical domain. In 2016 Radiology Informatics Laboratory Contour (RIL-Contour) was published [29]. RIL-Contour is an annotation tool for medical image datasets. Deep Learning algorithms support it to label images for Deep Learning research. The tool most similar to ours is Endometriosis Annotation Tool [30]. The software, developed by a group of developers and gynecologists, is a web-based annotation tool for endoscopy videos. In addition to the classic functions such as video controls, screenshots, or manual labeling of the images, the option of selecting between different endometriosis types is also offered here. Nevertheless, most of these medical annotation tools are not suitable for our comparison as they only work with images or are too specialized. The most suitable would be Endometriosis Annotation Tool, but the application is focused on specific annotations for surgery and those not allow the creation of bounding box annotations which are crucial for our gastroenterological annotations. Therefore, we choose a common, well-known state-of-the-art tool CVAT, for our comparison.

\section{Results}

This section presents the results of our introduced tool FastCAT and compares it to the well-known state-of-the-art annotation tool CVAT. We start by introducing our data acquisition and experimental setup. We show our results of the non-expert annotators, which suggests that our tool outperforms the state-of-the-art tool CVAT. We further show how the semi-automated AI annotation affects the annotation speed. Finally, we show our results of the expert annotator, which underline the time advantage using our tool.

\section{Data acquisition and experimental set up}

For our evaluation, we used two data sets: The GIANA data set and our data set created at a German clinic called "University Hospital Würzburg" [2]. The GIANA

${ }^{[2]}$ https://www.ukw.de/en 
dataset is openly accessible ${ }^{[3]}$ [31]. It is the first polyp dataset published, which includes videos. Former open-source datasets like CVC clinic database [32] or ETISLaribPolypDB [33] only provide single images. The GIANA dataset consists of 18 annotated polyp sequences. It is a standard dataset that has been used before for model benchmarking in different publications $[34,35,36]$. Therefore, we can reliably use it for evaluating the quality of our results. On average, the data set has 714 frames per video. According to their references, all annotations are done by expert gastroenterologists. We randomly selected two videos from the 18 available ones in GIANA for our evaluation, which turned out to be videos number 8 and 16 .

Our data set is composed of an additional 8 videos. These videos include full colonoscopies and therefore have to be filtered first. For the filtering process, we used the method introduced in this paper. Furthermore, we contacted an expert gastroenterologist from the University Hospital Würzburg for the expert annotation. Since the expert annotation time of gastroenterologists is very costly and difficult to obtain, we could only manage to receive the work of one expert. In a second process, the expert annotator selects the part of the video, including polyps, as explained in section Methods. However, since this annotation process is not yet completed, we can only evaluate the improvement in annotation speed and not the annotation quality with our dataset.

For the study, all participants receive ten videos for polyp annotation. The videos are randomly selected and then given to the participants. For our preliminary evaluation, ten test subjects are instructed to use our annotation tool and the stateof-the-art annotation tool CVAT. Finally, all non-expert annotators receive our software FastCAT and a java tool for measuring the time. The expert annotator starts with annotation, as explained in Methods. He annotates Paris classification [37], the size of the polyp, and its location. Additionally, the expert annotates the start and end frame of the polyp and one box for the non-expert annotators. Afterwards, the AI calculates predictions on these images. The results of the AI are given to the non-expert annotators, who then only correct the predicted boxes. The test subjects in this experiment are students from computer science, medical assistance, and medical secretary. All non-expert annotators are instructed to annotate the polyp frames as fast and as accurately as they can.

\section{Results of the non-expert annotators}

We evaluated the tool with 10 different gastroenterological videos containing full colonoscopies. The results are shown in table 2 and in table 3 . As mentioned previously, we only evaluate the quality of the annotation in two videos from the openly accessible GIANA dataset. The quality evaluation is done via the F1-score. The F1-score describes the harmonic mean of precision and recall as show in following equations:

$$
\begin{gathered}
\text { Precision }=\frac{T P}{T P+F P} \quad \text { Recall }=\frac{T P}{T P+F N} \\
F_{1}=\frac{2 * \text { Precision } * \text { Recall }}{\text { Precision }+ \text { Recall }}=\frac{2 * T P}{2 * T P+F P+F N}
\end{gathered}
$$

${ }^{[3]}$ https://endovissub2017-giana.grand-challenge.org 
Table 2 Comparison of FastCAT and CVAT by video. This table shows our comparison of the well-known CVAT annotation tool to our new annotation tool FastCAT in terms of annotation speed. Videos 1 and 2 are open source and annotated. Video 3 - 10 are from the University Hospital Würzburg.

\begin{tabular}{l|cc|cc||ccc}
\hline \hline & \multicolumn{2}{|c|}{ Speed (SPF) } & \multicolumn{2}{c||}{ Total time (min) } & \multicolumn{3}{c}{ Video information } \\
\hline & CVAT & Ours & CVAT & Ours & Frames & Polyps & Framesize \\
\hline Video 1 & 3.79 & $\mathbf{1 . 7 5}$ & 23.43 & $\mathbf{1 0 . 8 2}$ & 371 & 1 & $384 \times 288$ \\
Video 2 & 4.39 & $\mathbf{2 . 4 9}$ & 32.85 & $\mathbf{1 8 . 6 3}$ & 449 & 1 & $384 \times 288$ \\
Video 3 & 2.82 & $\mathbf{1 . 4 2}$ & 60.11 & $\mathbf{3 0 . 2 7}$ & 1279 & 1 & $898 \times 720$ \\
Video 4 & 4.09 & $\mathbf{2 . 0 0}$ & 56.85 & $\mathbf{2 7 . 8 0}$ & 834 & 1 & $898 \times 720$ \\
Video 5 & 4.57 & $\mathbf{2 . 3 9}$ & 53.24 & $\mathbf{2 7 . 8 4}$ & 699 & 2 & $898 \times 720$ \\
Video 6 & 1.66 & $\mathbf{0 . 6 1}$ & 18.01 & $\mathbf{6 . 6 2}$ & 651 & 1 & $898 \times 720$ \\
Video 7 & 1.70 & $\mathbf{0 . 6 4}$ & 11.22 & $\mathbf{4 . 2 2}$ & 396 & 1 & $898 \times 720$ \\
Video 8 & 1.55 & $\mathbf{0 . 7 6}$ & 34.13 & $\mathbf{1 6 . 7 3}$ & 1321 & 2 & $898 \times 720$ \\
Video 9 & 1.87 & $\mathbf{0 . 8 8}$ & 34.91 & $\mathbf{1 6 . 4 3}$ & 1120 & 1 & $898 \times 720$ \\
Video 10 & 2.74 & $\mathbf{0 . 9 2}$ & 77.68 & $\mathbf{2 6 . 0 8}$ & 1701 & 4 & $898 \times 720$ \\
\hline Mean & 2.92 & $\mathbf{1 . 3 9}$ & 40.24 & $\mathbf{1 8 . 5 4}$ & 882 & 1.5 & $795 \times 633$ \\
\hline \hline
\end{tabular}

Table 3 Comparison of FastCAT and CVAT by user. This table shows our comparison of the well-known CVAT annotation tool to our new annotation tool FastCAT in terms of quality of annotation and annotation speed. The quality metric is the F1-score. We count a TP if the drawn box matches the ground truth box more than $70 \%$.

\begin{tabular}{l|cc|cc|cc||c}
\hline \hline & \multicolumn{2}{|c|}{ Quality (\%) } & \multicolumn{2}{|c|}{ Speed (SPF) } & \multicolumn{2}{|c||}{ Total time (min) } & Medical experience \\
\hline & CVAT & Ours & CVAT & Ours & CVAT & Ours & \\
\hline User 1 & 99.30 & $\mathbf{9 9 . 5 0}$ & 7.33 & $\mathbf{3 . 7 1}$ & 48.78 & $\mathbf{2 5 . 3 0}$ & low \\
User 2 & 98.85 & $\mathbf{9 8 . 9 0}$ & 3.47 & $\mathbf{1 . 8 8}$ & 23.38 & $\mathbf{1 3 . 7 0}$ & low \\
User 3 & 97.97 & $\mathbf{9 8 . 5 1}$ & 4.59 & $\mathbf{1 . 5 3}$ & 31.28 & $\mathbf{1 1 . 1 7}$ & low \\
User 4 & 98.93 & $\mathbf{9 9 . 7 5}$ & 5.12 & $\mathbf{2 . 5 7}$ & 33.96 & $\mathbf{1 6 . 5 3}$ & middle \\
User 5 & 98.53 & $\mathbf{9 8 . 8 3}$ & 5.41 & $\mathbf{2 . 4 9}$ & 37.00 & $\mathbf{1 8 . 1 0}$ & middle \\
User 6 & 98.52 & $\mathbf{9 9 . 2 3}$ & 4.04 & $\mathbf{3 . 2 4}$ & 27.90 & $\mathbf{2 4 . 9 5}$ & low \\
User 7 & $\mathbf{9 9 . 4 5}$ & 99.30 & 5.20 & $\mathbf{2 . 7 0}$ & 35.01 & $\mathbf{2 1 . 2 8}$ & middle \\
User 8 & $\mathbf{9 9 . 3 5}$ & 99.08 & 5.25 & $\mathbf{2 . 8 6}$ & 33.90 & $\mathbf{1 9 . 5 7}$ & low \\
User 9 & $\mathbf{9 9 . 1 2}$ & 98.54 & 4.12 & $\mathbf{2 . 2 5}$ & 27.12 & $\mathbf{1 4 . 9 9}$ & low \\
User 10 & 98.93 & $\mathbf{9 9 . 4 8}$ & 5.63 & $\mathbf{2 . 7 6}$ & 37.53 & $\mathbf{1 9 . 8 9}$ & low \\
\hline Mean & 98.98 & $\mathbf{9 9 . 0 3}$ & 5.79 & $\mathbf{2 . 9 3}$ & 33.59 & $\mathbf{1 8 . 5 5}$ & low \\
\hline \hline
\end{tabular}

We count an annotation as true positive (TP) if the boxes of our annotators and the boxes from the GIANA dataset have an overlap of at least 70\%. Our experiments showed high variability between individual experts. We, therefore, concluded that a higher overlap is not attainable. Hence, to ensure reasonable accuracy, we choose an overlap of $70 \%$ which has been used in previous studies [38, 39, 40]. To determine annotation speed, we first measure the speed of the non-expert annotators in seconds per frame (SPF). On average, our annotators take 2.93 seconds for annotating one image while maintaining a slight advantage in annotation quality. Overall, our semiautomated tool's annotation speed is almost $2 \mathrm{x}$ faster than the CVAT annotation tool, with 5.79 seconds per image. In addition, we evaluate the average time nonexpert annotators spend annotating an entire video. The average video takes 18.55 minutes to annotate. In comparison, using the CVAT tool takes 40.24 minutes on average per video. Due to some faulty prediction results of the neural network, the annotators sometimes delete boxes and draw new boxes as some polyps may be hard to find for the CNN. This leads to higher annotation time in the case where polyps are mispredicted. Nevertheless, our tool is self-learning, and increasing amounts of high-quality annotations improve the prediction quality of the CNN. This, in turn, speeds up the annotation process further. We elaborate on this in detail in the 
following subsection. To include more information concerning the video data, we include the number of frames per video, the number of polyps per video, and each video's frame size. The videos provided by our clinic (Videos 3-10) have a higher resolution and a higher frame rate than videos gathered from different institutes. Overall the quality evaluation results show that almost similar annotation results to those of gastroenterology experts are achieved. For speed, our tool outperforms the CVAT tool in any video. In two videos, our tool is more than twice as fast as the CVAT tool.

\section{Learning process of the non-expert annotators}

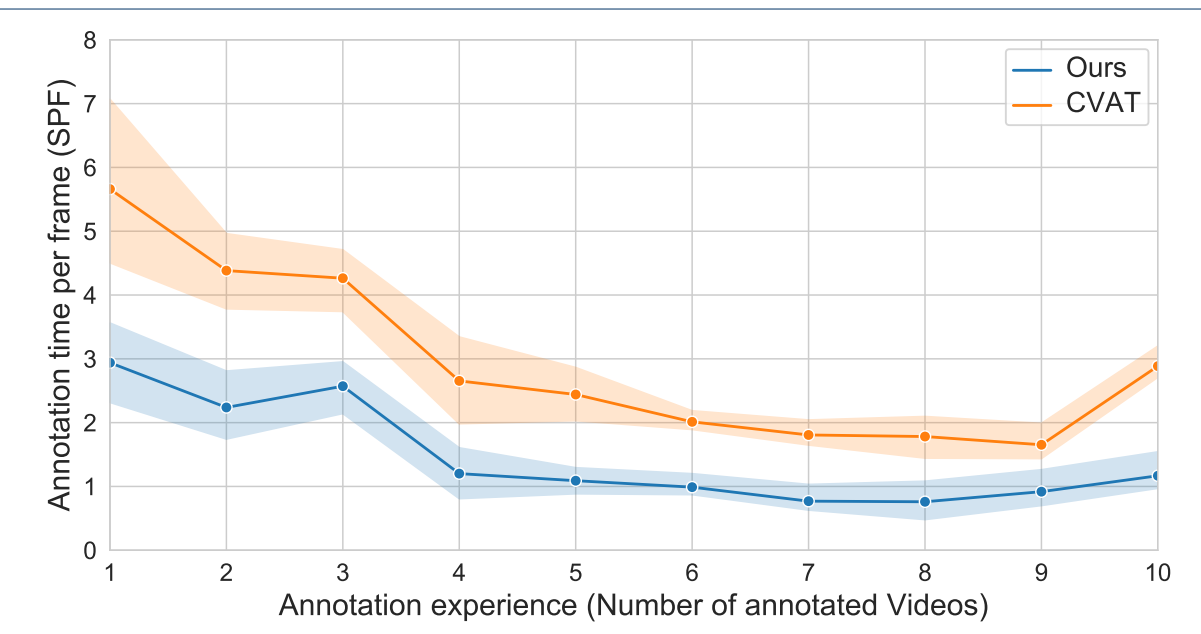

Figure 1 Learning process of the non-expert annotators. The figure shows the speed of the annotator in seconds per frame (SPF) over the annotation experience measured by the total number of annotated videos by that point for both our tool and CVAT.

Figure 1 shows the learning process of the non-expert annotators, in blue using our tool and in orange using CVAT. The figure shows that the annotation of the first videos takes longer than annotating the subsequent ones since the subject has to get to know the software and needs to adjust the software to his preferences. Therefore, annotation speed using both tools improves by further usage, and both tools feature a similar learning curve. However, this learning process slows down after the annotation of about 4 to 5 videos. After this amount of videos, annotators are well accustomed to the software and can competently use most features. In addition, figure 1 shows that this learning process is faster using our tool in comparison to the CVAT tool. This may be due to the information provided before use, the calculation we built directly into the software, and our user-friendly environment. Besides all, the CVAT software also shows excellent progress in learning worth mentioning. We can even see annotators who use any of the two tools more frequently further improve their annotation speed up to 9 videos. However, after 8 to 9 videos, the annotation speed decreases. This may be due to two repetitions of the same process that may bore the subject and, therefore, decrease annotation speed. Our data show that this effect is more prominent for CVAT than for our tool. 


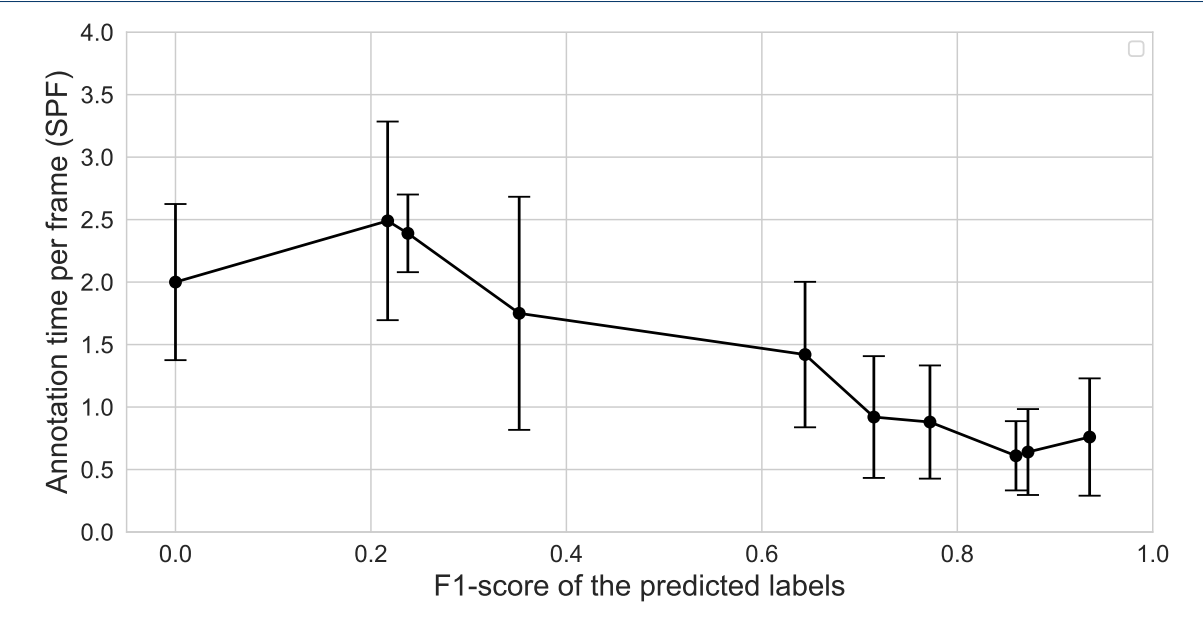

Figure 2 Effect of model performance on annotation speed. Plotted are the speed of the annotators in seconds per frame over the model performance given by its F1-score on a video by video basis, where the model used for prediction is the same for each video. Every point is computed as the average over all annotators.

\section{Impact of polyp pre-annotations}

To further analyze the improvements in our framework, we investigate the impact of polyp detection on the annotation speed. We compare the final annotated videos with the predictions done during the investigated videos. For ten videos, we calculated the F1-score based on the analysis above. A higher F1-score implicates more detected polyps with less false positive detection. Then, we rank the videos according to their F1-score and display the annotation speed in seconds per frame (SPF), shown in Figure 2. Overall, a high F1-score leads to a faster annotation speed. Nevertheless, as seen in figure 2 if the F1-score is low, the annotation speed at times is faster without any predictions, e.g., from 0.2 to 0.4 . Furthermore, low F1-scores show a higher standard deviation in the labeling speed. This means that with a higher F1-score, the variance of the participants' labeling speed decreases and therefore the overall performance is increased. Furthermore, we emphasize that continuing the annotation process and retraining the system detection results will increase, and therefore, the annotation speed will increase. 
Table 4 Comparison of CVAT and FastCAT. The tables show the reduction of annotation time of the domain expert. Tgac stands for the time gained compared to annotation with CVAT and is the reduction of workload in $\%$.

\begin{tabular}{l|cc|c|ccc}
\hline \hline & \multicolumn{2}{|c|}{ Total time (min) } & Tgac (\%) & \multicolumn{3}{c}{ Video information } \\
\hline & Ours & CVAT & & Length (min) & Freezes & Polyps \\
\hline Video 3 & 0.51 & 60.11 & 99.15 & 15.76 & 2 & 1 \\
Video 4 & 0.67 & 56.85 & 98.82 & 17.70 & 6 & 1 \\
Video 5 & 0.88 & 53.24 & 98.35 & 23.12 & 4 & 2 \\
Video 6 & 0.54 & 18.01 & 97.00 & 6.30 & 2 & 1 \\
Video 7 & 0.91 & 11.22 & 95.36 & 13.05 & 5 & 1 \\
Video 8 & 1.94 & 34.13 & 94.31 & 27.67 & 13 & 2 \\
Video 9 & 2.05 & 34.91 & 94.13 & 20.53 & 4 & 1 \\
Video 10 & 2.19 & 77.68 & 97.18 & 24.36 & 15 & 4 \\
\hline Mean & 1.21 & 43.26 & $\mathbf{9 6 . 7 9}$ & 18.56 & 6.38 & 1.62 \\
\hline \hline
\end{tabular}

\section{Results of the expert annotator}

This subsection demonstrates the value of the tool for domain expert annotation. As domain experts are very costly, we only had a single expert available for our study. Therefore, our evaluation between domain experts could not be done quantitatively. Nevertheless, we can qualitatively compare the amount of time a domain expert annotates our collected colonoscopies. This is shown in table 4. On average, our gastroenterologist spends 1.34 minutes on a colonoscopy. Our final results show that we achieve qualitatively similar results to the GIANA dataset annotation. The expert annotator only takes 0.5 to 1 minute per video using our method, while taking at least 10-80 minutes per video using the CVAT software. Therefore, we can reduce the amount of time a domain expert has to spend on annotation by 96.79 $\%$ or by a factor of 20 . This reduction is primarily due to expert and non-expert annotation structure, which reduces the expert's effort tremendously.

\section{Discussion}

By implementing a novel workflow consisting of both algorithmic and manual annotation steps, we developed a tool that significantly reduces the workload of expert annotators and improves overall annotation speed compared to existing tools. In this section, we highlight and discuss the impacts of our study, show the limitation of our presented work and propose new approaches to advance our study further.

\section{Key features and findings}

Our results show that by pre-selecting relevant frames using a combination of our freeze-frame detection algorithm and further, low-demand expert annotations and by using AI-predictions for bounding box suggestions, we significantly increase the annotation speed while maintaining and even increasing annotation accuracy (see table 2 and 3). It is important to note that this improvement is not due to more annotation experience with one tool over the other since the test annotators used the tools in an alternating fashion with random video order. Figure 1 further stresses this fact by showing a similar learning curve for both tools, with our tool being shifted down to shorter annotation times. In both cases, the annotation experience (i.e., adjustment to the tool) increases up to around seven videos or 10000 annotated frames. The annotation speed first saturates and then increases again, possibly due to a human exhaustion effect of doing the same task for an extended duration [41]. 
Additionally, we inspected the effect of the prediction performance on the annotation speed. As shown in figure 2, there is a clear trend towards faster annotation time with better model performance. The annotator works faster if the suggested bounding boxes are already in the correct location or only need to be adjusted slightly by drag and drop. If the predictions are wrong, the annotator needs to move the boxes further, perhaps readjust the size more, or even delete boxes or create new ones. However, the model improvement saturates at an F1-score of around 0.8 , where better model performance does not equate to faster annotation speed. Additionally, the range of error is much more significant for the worse performing videos, so this point warrants further inspection in future studies. Nevertheless, it is apparent here that a model only needs to be good enough instead of perfect to improve annotation speed significantly.

Finally, the results in table 3 suggest that medical experience does not affect either the annotation speed or performance. The frame detection algorithm combined with the expert frame annotations and our model's pre-detection provides enough feasibility for the non-experts to adjust the suggested annotations fast and accurately regardless of experience. However, it should be noted that the range of speeds across our subjects is more stable for middle experience annotators than low experience ones.

All in all, our tool significantly improves the annotation workflow, specifically in the domain of gastroenterology, where specialized tools are scarce. The annotation speed is more than doubled while keeping the same accuracy as other state-of-theart tools and keeping the cost for expert annotators low.

\section{Limitations of the study}

In this subsection, we will shortly discuss the limitations of our analysis and provide an outlook for future studies.

First of all, we did not consider the difficulty of the video when analyzing annotation time. Some videos contain more and harder to detect polyps and thus provide a bigger challenge for both the pre-detection algorithm and the annotator. The effect of video difficulty directly correlates to the model performance in figure 2 , where the standard error for low-F1 videos is much higher compared to the better ones. Some annotators can efficiently deal with false predictions, while others have more difficulties with those. Additionally, the total annotation time was measured from beginning to end for a video. While the applet we provided for the annotators includes a pause button, minor deviations, like checking their phone, are not removed from our total time measured. These statistical deviations could be removed by dividing the videos into difficulty categories and analyzing each category separately. We need more data or more test annotators, where small statistical outliers should be averaged out.

Additionally, with only three medical assistants and seven non-experts, we need further tests to see if medical experience significantly affects annotation time and quality. As discussed above, table 3 suggests that medium experience annotators work more consistently, whereas low experience ones can be both faster and slower than the medical assistants. These findings can be examined further in future studies with more annotators from various backgrounds, especially those with high medical experience. 
Finally, we only indirectly measured the effect of bounding box pre-detection, where our subjects had no pre-detection for CVAT and suggestions with our tool. Thus, the improvement in annotation speed could also be due to our tool simply being easier to use and having a better UI than CVAT. For future analysis, we intend to have the test subjects annotate videos twice, once with bounding box suggestions and once without. However, both times they will use our tool. This way, we will be able to analyze the effect of the pre-detection directly.

\section{Limitations of the tool and future improvements}

While our freeze frame detection algorithm is specific to the domain of gastroenterology, the specific method for detecting relevant frames can be exchanged for a function more suited to the annotators' domain. Additionally, while we only utilized the tool for polyp detection, it can be easily extended to feature more than one pathology, like diverticulum or inflammation. Since frame-wide annotations are separate from bounding boxes, this can also be used for standard image classification tasks and pathologies that are hard to confine to a bounding box area.

Additionally, within the medical domain, we plan to implement a feature for automatically detecting gastroenterological tools. When the acting doctor detects a suspicious polyp or other, they often remove them during the examination. The tools will then be visible on screen and are an indicator of pathology. Hence, the tool detection can be used as an algorithm to detect relevant frames within the videos.

The pre-detection algorithm itself is also not limited to our deep learning model trained on polyps but can be exchanged easily for a model more suited to the user's task.

The algorithm used for tracking objects across several frames is currently limited by the implemented standard object trackers above. These trackers are standard tools that often lose the object and have much room for improvement. While we provide an option for resetting the trackers, we intend to implement state-of-the-art video detection algorithms in the future to fully utilize this feature [42, 43].

\section{Conclusion}

In this paper, we introduce a framework for fast expert annotation, which reduces the working amount of the domain expert by a factor of 20 while retaining very high annotation quality. We publish open-source software for annotation in the gastroenterological domain and beyond. This includes two views, one for expert annotation and one for non-expert annotation. We incorporate a semi-automated annotation process in the software, which reduces time spend on annotation and further enhances the annotation quality. Our results suggest that our tool enhances the medical especially endoscopic image and video annotation, tremendously. We not only reduce the time spend on annotation by the domain expert but also the overall effort. 


\section{Methods}

In this section, we explain our framework and software for fast semi-automated machine learning video annotation. The whole framework is illustrated in figure 1 . The annotation process is split between at least two people. At first, an expert reviews the video and annotates a few video frames to verify the object's annotations. In a second step, a non-expert has visual confirmation of the given object and can annotate all following and preceding images with AI assistance. To annotate individual frames, all frames of the video must be extracted. Relevant scenes can be selected by saving individual frames. This prevents the expert from reviewing the entire video every single time. After the expert has finished, relevant frames will be selected and passed on to an AI model. This information allows the AI model to detect and mark the desired object on all following and preceding frames with an annotation. Therefore, the non-expert can adjust and modify the AI predictions and export the results, which can then be used to train the AI model.

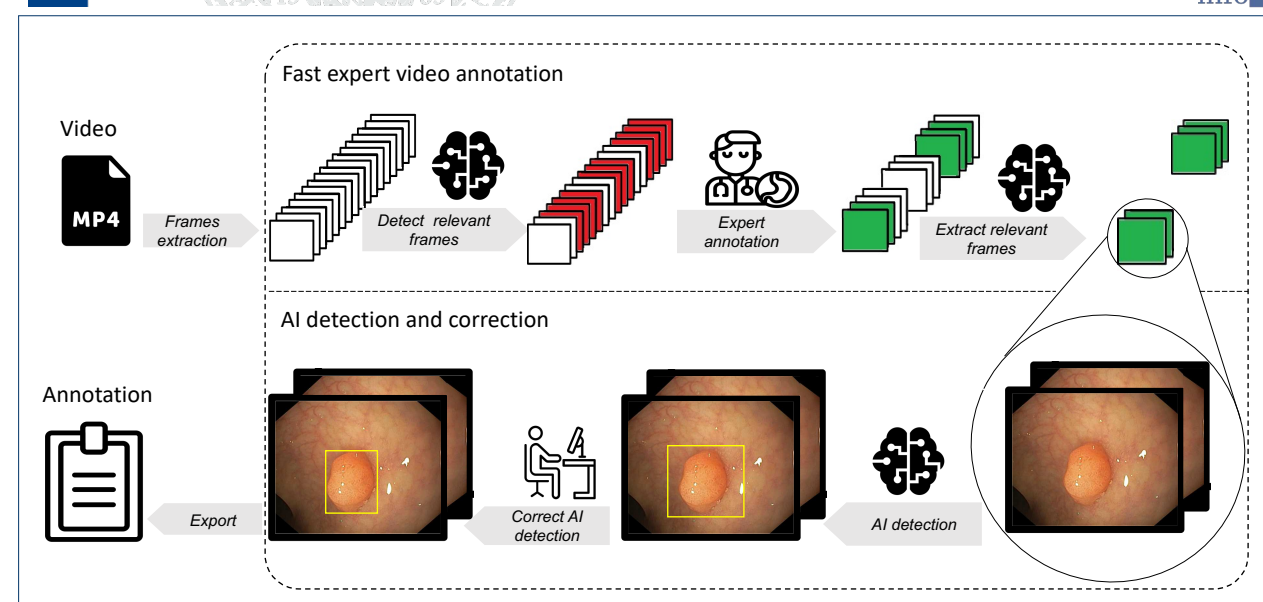

Figure 3 Annotation framework for fast domain expert labeling supported by an automated machine learning pre-labeling.

Input

To annotate individual video frames, the program must have access to all frames of the video. If annotated frames already exist, the program can recognize this; otherwise, it will extract all frames from the video and save them into a separate folder. Relevant frames can be marked manually or inferred automatically. To mark the frames manually, enter frame numbers or timestamps in the program. In the context of our polyp detection task, we created a script that detects when the recording freezes and marks these frames as relevant. A video freeze is caused by photos taken of suspicious polyps that are taken during the examination. Therefore, these parts of the video are most relevant for the expert. This reduces the expert's workload since he does not have to review the entire video but can quickly jump to the relevant parts of the video. The extraction is done by using the OpenCV library. 


\section{Detect relevant frames}

We denote all frames that assist the expert in finding critical parts of the video as superframes. Such frames can be detected automatically or entered manually by a frame number or timestamp. During a colonoscopic or gastroscopic examination, when the acting doctor detects a polyp (or similar), they freeze the video feed for a second and capture a photo of the polyp. Hence, for our task (annotation in gastroenterology), we automatically detect all positions in which a video shows the same frames for a short time, i.e., where the video is frozen for a few frames. Overall, within our implementation, we call such a position a superframe. The detailed explanation for detecting those freeze frames is shown in algorithm 1.

In order to discover those freezes automatically, we extract all frames from the video using OpenCV [44]. Afterwards, we compare each frame to its next frame. This is done by computing the difference in pixel values of both frames, converting it into the HSV color space, and calculating an average norm by using the saturation and value dimension of the HSV color model. A low average norm means that both frames are almost identical; hence a freeze could have happened. We save a batch of ten comparisons for a higher certainty and take an average of the ten last comparisons (similar to a moving average). If the average value falls below a certain threshold, we define the current frame as the start of a freeze. The end of a freezing phase is determined if the average value exceeds another defined threshold. This algorithm has high robustness and consistency as it rarely misses a freeze or creates a false detection.

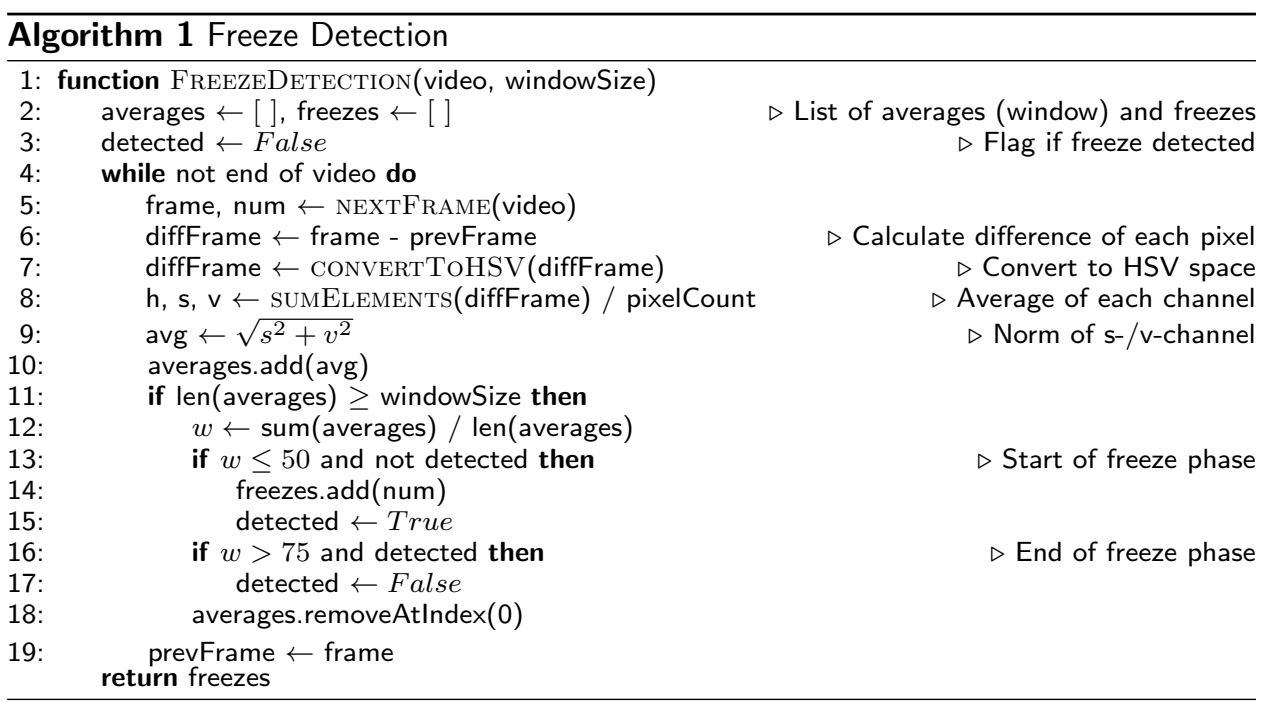

\section{Expert View}

We refer to this part of the program Video Review, as the expert reviews the video to find polyps. For the expert to perform their task, they require the examination video, all individual video frames, and a set of relevant frame numbers, e.g. superframes. The video allows the expert to review the performed examination and get an overview of the presented situation to diagnose polyps correctly. All extracted video frames are necessary to be able to access and annotate individual frames. 
Lastly, a set of relevant frame numbers is given to the expert to jump to relevant video parts quickly. This led to a solution that provides the expert with two different viewpoints: (1) video player and (2) frame viewer. To enable fast and smooth transition between both viewpoints, it is possible to switch at any point in time from the current video time stamp $t$ to the corresponding video frame $f$ and vice versa. This is done by a simple calculation based on the frames per second (FPS) of the video and the current timestamp in milliseconds: $f=\frac{t[\mathrm{~ms}] \cdot \mathrm{FPS}[1 / \mathrm{s}]}{1000}$.

It is possible to look at individual video frames within the frame viewer, assign classes to these frames, and annotate polyps within those frames. The class assignment is done through superframes, where each frame to which a class is assigned will be associated with a previously selected superframe. The second task, frame annotation, is independent of a class assignment and annotates the polyps within a frame with a bounding box that encloses the polyp. This primarily serves as an indication for non-experts to get visual information about the polyp that can be seen in the following/subsequent frames.

We use classes to mark frames if there is a polyp in the picture; we use these classes to mark relevant frames for the following annotation process by a non-expert. Two different approaches can be used to assign classes to frames. A range of frames is defined in the first approach by assigning start and end classes to two different frames. Consequential, all frames in between belong to the same class. The tool is also capable of assigning classes to each frame individually. The changes within video frames are small; therefore, many consecutive frames must be annotated with the same class. To make this process less time-consuming, the program allows the expert to go through a sequence of frames quickly and smoothly while classifying them by keeping a key pressed. However, mostly the assignment of start and end classes is faster and prefered.

Because all frames are mostly stored on an $\mathrm{HDD} / \mathrm{SSD}$, the loading latency is a performance bottleneck. We implemented a pre-loading queue that loads and stores the upcoming frames into the RAM to achieve fast loading times. This allows to display and assign frames with low latency. To prevent the queue from emptying rapidly, which causes high loading latency, we need to control the queue access times between two frames. Therefore, we use a capacity-dependent polynomial function to calculate a pausing time between frames: $\mathrm{ms}=50 \cdot(1-\text { capacity })^{2.75}$. A full queue shortens the waiting time to $0 \mathrm{~ms}$, while an empty queue leads to a $50 \mathrm{~ms}$ waiting time. This method combines fluent viewing and class assigning while providing enough time in the background to load new frames continuously.

Since the basic information about the presence of a polyp on an image is not sufficient for non-experts, and we want to ensure high-quality annotations, the expert has to annotate samples of all discovered polyps. This will provide visual information of the polyp to non-experts, allowing them to identify these polyps in all following and preceding images correctly. Scenes in which polyps are difficult to identify due to perspective changes and other impairments should also be exemplary annotated by experts to provide as much information as possible to non-experts.

As we can see in figure 4 on the left side, the program lists all detected freeze frames. The list below shows all frames that belong to the selected freeze-frame and were annotated with specific classes, e.g., polyp type. Independent from the 
hierarchical structure above, we display all annotations that belong to the current frame in a list and on top of the image. In the lower part of the view, navigation controls skip a certain amount of frames or jump directly to a specific frame. The annotator can also leave a note to each frame if necessary or delete certain classes from the frame.

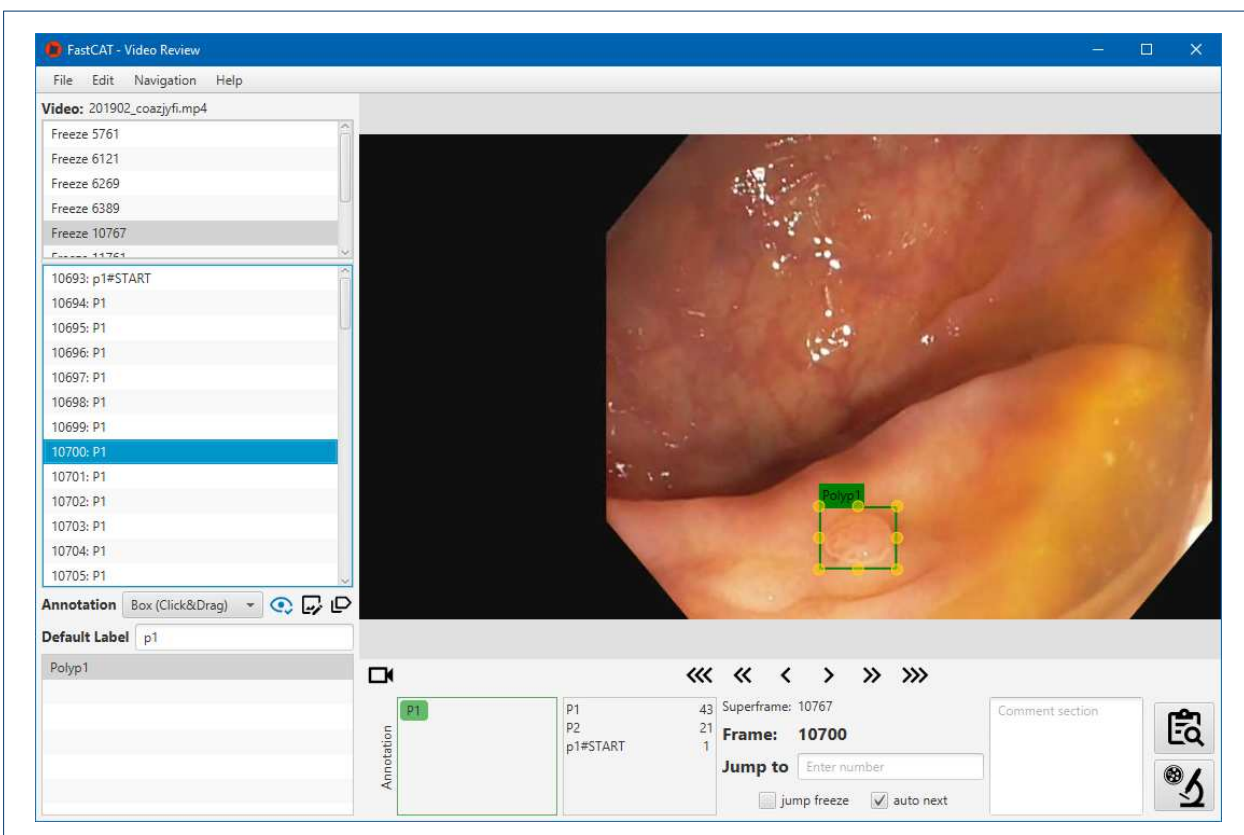

Figure 4 Video Review UI. The figure shows the list of freeze frames, the corresponding child frames, and annotations within the image on the right side. In the bottom part of the view, the user can insert comments, open reports, delete classes, and see all individual classes.

\section{Semi-automated polyp prelabeling}

The prediction of polyps is made by an object detection model that was trained to detect polyps. The task of polyp detection is a combination of localizing and classifying an identified polyp. With this method, we aim for a fast AI-assisted annotation process for non-experts. Since every team has a different application, we distinguish between offline and online polyp prediction.

With an offline polyp prediction approach, we eliminate the need for high-end hardware for each user who uses AI assistance for fast annotation. The prediction is made by an external machine that is capable of running an AI model. With this approach, the extracted relevant frames are passed to this machine, generating a tool-specific JSON file that is then passed to the non-expert for further inspection.

As online polyp prediction, we define the performance of polyp detection locally on the machine of the annotator. Therefore, the machine on which our tool is executed must have the necessary hardware and software installed to run the detection model. As there are different frameworks and deep learning networks, we need a unified interface to address all these different requirements. We decided to use Docker ${ }^{[4]}$ for this task. Docker uses isolated environments called containers. These containers

${ }^{[4]}$ https://docker.com 
only carry the necessary libraries and frameworks to execute a program. By creating special containers for each model, we can run a prediction independent of our tool and its environment. Containers are built from templates called images, which can be published and shared between users. Therefore, it is possible to create a repository of different models and prediction objectives. Because a container shuts down after every prediction, it must reload the model for the next prediction. To counteract this, we run a web server inside the container and communicate to the model via HTTP. This ensures that a model does not have to reload after every prediction and provides a universal and model-independent communication interface. With this setup, the user can trigger a single prediction or run a series of predictions in the background.

As we have already stated, we use HTTP for our communication. This gives room for a hybrid solution, allowing predictions on an external server while retaining the user's control. This combines the advantages of the external and local approaches, where the user is not required to have expensive hardware, nor is it necessary to have a separate, time-consuming prediction step.

\section{Non-Expert Annotation}

With the help of AI, it is possible to annotate a large number of frames quickly and easily. However, this method does not ensure the correctness of the predicted annotations. For this reason, these annotations must be checked and modified if necessary. Non-experts can check these predictions or create new annotations with the help of verified example annotations from the expert and the indication in which frame a polyp is visible. Besides, the AI-assisted support of our tool provides annotation duplication across several frames and object tracking functionality which speeds up the annotation process.

As mentioned in section Semi-automated polyp prelabeling our tool supports the integration of AI detection. It can trigger a single prediction or make predictions on the following frames in the background. This enables the user to immediately annotate the remaining frames without waiting for the external prediction process to finish.

Another helpful feature is the duplication of annotations. Sometimes, only subtle movements occur in polyp examination videos, causing a series of frames to only show minuscule changes. This feature allows the non-expert to use the bounding boxes of the previous frame and only make minor adjustments while navigating through the images. Re-positioning an existing bounding box requires less time than creating an entirely new box with a click and drag motion.

Our last feature uses object tracking to track polyps throughout consecutive frames. This avoids the manual creation of bounding boxes for each video frame, especially in sequences where an object's visual and spatial transition between two frames is non-disruptive. For this task, we used trackers available in the OpenCV library. Within the intestine, special conditions are usually present. First, the nature of colonoscopies leads to unsteady camera movement. Second, the color of polyps is often similar to the surrounding intestinal wall, which can make them hard to recognize. This can compromise the performance of the tracker and deteriorate polyp tracking. Given the fact that the annotation process requires a user to operate the 
tool and, therefore, the tracker does not need to track polyps fully automatically, we added two options to reset the tracker. This is described in more detail in the next section.

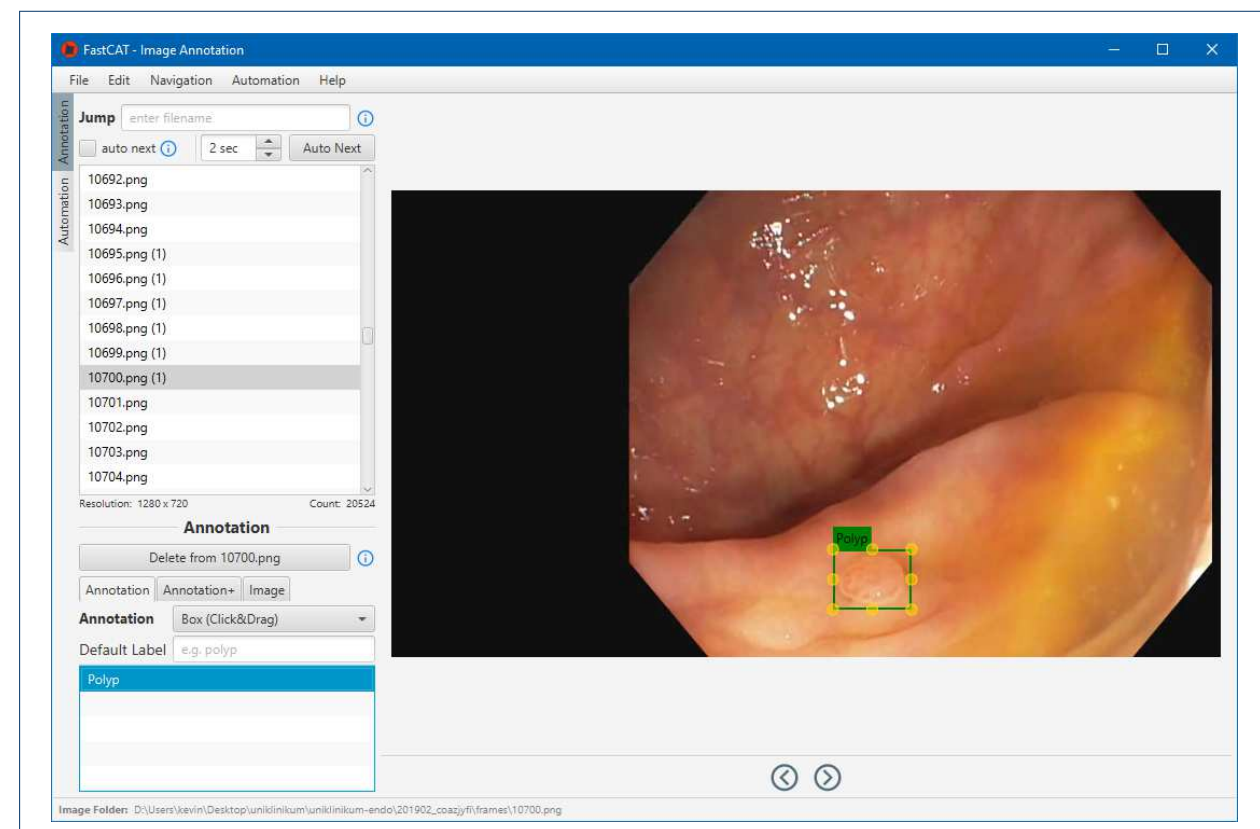

Figure 5 Image Annotation UI. The figure shows a list of all available images on the left with labeling functionality for a specific annotation and the whole image. The image to be annotated is displayed on the right.

\section{Object Trackers}

As described in section Non-Expert Annotation our tool has object tracking functionality. It assists in tracking an object across multiple frames. For our tool, we implement six of the available trackers in the OpenCV library [44]. In the following, we give a short description of the available trackers:

- Boosting: It is using an online version of AdaBoost to train the classifier. Therefore, the tracking is viewed as a binary classification problem, and negative samples of the same size are extracted from the surrounding background. It can update features of the classifier during tracking to adjust to appearance changes [45].

- MIL: Multiple Instance Learning uses a similar approach as Boosting and extracts positive samples from the immediate neighborhood of the object. The set of samples is put into a bag. A bag is positive when it contains at least one positive example, and the learning algorithm has to the inference which is the correct sample within a positive bag [46].

- KCF: Kernelized Correlation Filter uses the same basic idea as MIL, but instead of sampling a handful of random samples, it trains a classifier with all samples. It exploits the mathematical properties of circulant matrices to make tracking faster and better [47].

- CSRT: CSRT uses discriminative correlation filters (CDF) with channel and spatial reliability concepts. The correlation filter finds similarities between the 
two frames. The spatial reliability map restricts the filter to suitable parts of the image. Scores estimate the channel reliability to weight features. [48] In addition, it is worth mentioning that rapid movements are not handled well by trackers that use CDF [49].

- Median Flow: Median Flow tracks points of the object forward and backward in time. Thereby, two trajectories are measured, and an error between both trajectories is estimated. By filtering out high error points, the algorithm tracks the object with all remaining points. [50] It is best applicable for smooth and predictable movements [51].

- MOSSE: Minimum Output Sum of Squared Error is an adaptive correlation filter robust to light variation, scale, post, and deformations. It applies a correlation filter to detect the object in new frames. It works only with grayscale images, and colored images will be converted internally [52].

- TLD: TLD decomposes a long-term tracking task into tracking, learning, and detection. The tracker is responsible for tracking the object across the frames. The detector finds the object within a frame and corrects the tracker if necessary, and the learning part of the algorithm estimates the error of the detector and adjusts it accordingly [53].

An object tracker is designed to follow an object over a sequence of frames by locating its position in every frame. Each tracker uses different strategies and methods to perform its task. It can collect information such as orientation, area, or the shape of an object. However, also many potential distractions can occur during tracking that can make it hard to track the object. Distraction causes are, e.g., noisy images, unpredictable motion, changes in illumination, or complex shapes. As a result, the performance of different trackers can vary between different domains and datasets. For this reason, our tool allows the user to choose the best tracker for their task and dataset. Because trackers are primarily designed to track objects across many frames automatically, the tracker may generate less accurate bounding boxes over time or entirely lose track of the object. Since the tracking conditions for polyp detection are complex and our tool uses a semi-automated solution, we implemented two additional options for the annotation task.

By default, the tracker is initialized by placing a bounding box around an object that should be tracked. Consequently, the tracker will find the object on one consecutive frame and place a bounding box around it. We found that the tracker loses track of the initialized polyp with a high number of consecutive frames. Therefore we implemented options to reinitialize the tracker automatically. The first option reinitializes the tracker after every frame, giving the tracker the latest visual information of the polyp. The second option only initializes the tracker if the user changed the bounding box size. Both options ensure that the tracker has the latest visual information of the polyp since the user corrects misaligned bounding boxes.

\section{Output and Conversion}

We use JSON as our standard data format. The JSON prepared by the expert stores detected freeze frames with all corresponding frames that contain at least one class. Additionally, annotated frames are stored in the same file but independently from the class assignments. The resulting JSON from the expert annotation process serves as an intermediate output for further annotations. 
The non-expert produces the final output with all video annotations. This file contains a list of all images with at least one annotation. The tool produces a JSON with a structure designated to fit our needs. However, since different models require different data formats, we created a python script that converts our format into a delimiter-separated values (DSV) file format. Via a configuration file, the user can adjust the DSV file to its need, e.g., convert it into YOLO format. It is also possible to convert the DSV file back to our format. This enables seamless integration of different formats. In the future, further predefined formats can be added.

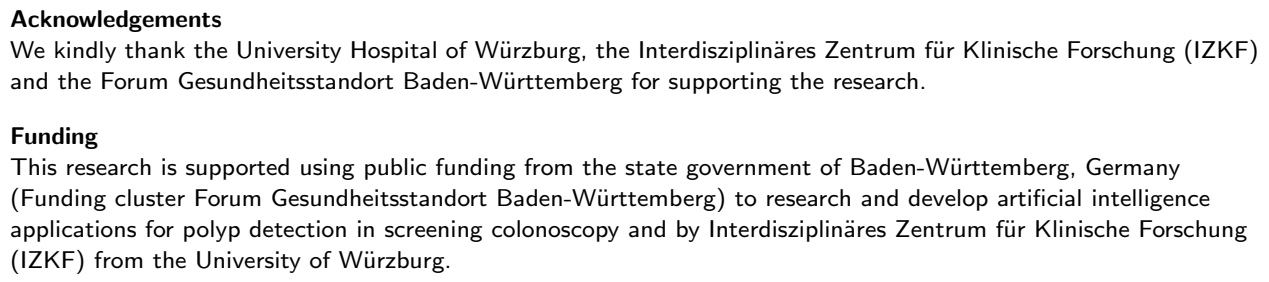

Availability of data and materials

The first dataset used for the analysis of this article is available in the GIANA challenge repository

(https://endovissub2017-giana.grand-challenge.org/). The second dataset used during the analysis is available from the corresponding author on reasonable request.

Ethics approval and consent to participate

Not applicable.

Competing interests

The authors declare that they have no competing interests.

Consent for publication

Not applicable.

Authors' contributions

AK implemented and coordinated the study, drafted the manuscript, and interpreted the data. KM and AK designed and implemented the software. $\mathrm{AH} 1$ and $\mathrm{KM}$ contributed to complete the manuscript. DF helped with the creation of the data. JT helped with the data preprocessing. FP, AH2 and WZ provided funding and reviewed the manuscript. All authors read and approved the final manuscript.

\section{Author details}

${ }^{1}$ Department of Artificial Intelligence and Knowledge Systems, Sanderring 2, 97070 Würzburg, Germany. ${ }^{2}$ Interventional and Experimental Endoscopy (InExEn), Department of Internal Medicine II, University Hospital Würzburg, Oberdürrbacher Straße 6, 97080 Würzburg, Germany. ${ }^{3}$ Department of Internal Medicine and Gastroenterology, Katharinenhospital, Kriegsbergstrasse 60, 70174 Stuttgart, Germany.

\section{References}

1. Miotto, R., Wang, F., Wang, S., Jiang, X., Dudley, J.T.: Deep learning for healthcare: review, opportunities and challenges. Briefings in bioinformatics 19(6), 1236-1246 (2018)

2. Litjens, G., Kooi, T., Bejnordi, B.E., Setio, A.A.A., Ciompi, F., Ghafoorian, M., Van Der Laak, J.A., Van Ginneken, B., Sánchez, C.I.: A survey on deep learning in medical image analysis. Medical image analysis 42, 60-88 (2017)

3. Erickson, B.J., Korfiatis, P., Akkus, Z., Kline, T.L.: Machine learning for medical imaging. Radiographics 37(2), 505-515 (2017)

4. Gunčar, G., Kukar, M., Notar, M., Brvar, M., Černelč, P., Notar, M., Notar, M.: An application of machine learning to haematological diagnosis. Scientific reports 8(1), 1-12 (2018)

5. Halama, N.: Machine learning for tissue diagnostics in oncology: brave new world. British Journal of Cancer 121(6), 431-433 (2019). doi:10.1038/s41416-019-0535-1

6. Kim, K.-J., Tagkopoulos, I.: Application of machine learning in rheumatic disease research. The Korean journal of internal medicine 34(4), 708 (2019)

7. Zerka, F., Barakat, S., Walsh, S., Bogowicz, M., Leijenaar, R.T., Jochems, A., Miraglio, B., Townend, D., Lambin, P.: Systematic review of privacy-preserving distributed machine learning from federated databases in health care. JCO clinical cancer informatics 4, 184-200 (2020)

8. Najafabadi, M.M., Villanustre, F., Khoshgoftaar, T.M., Seliya, N., Wald, R., Muharemagic, E.: Deep learning applications and challenges in big data analytics. Journal of big data 2(1), 1-21 (2015)

9. Chang, J.C., Amershi, S., Kamar, E.: Revolt: Collaborative crowdsourcing for labeling machine learning datasets. In: Proceedings of the $2017 \mathrm{CHI}$ Conference on Human Factors in Computing Systems, pp. 2334-2346 (2017) 
10. Vamathevan, J., Clark, D., Czodrowski, P., Dunham, I., Ferran, E., Lee, G., Li, B., Madabhushi, A., Shah, P., Spitzer, M., et al.: Applications of machine learning in drug discovery and development. Nature Reviews Drug Discovery 18(6), 463-477 (2019)

11. Webb, S.: Deep learning for biology. Nature 554(7693) (2018)

12. Bhagat, P.K., Choudhary, P.: Image annotation: Then and now. Image and Vision Computing 80 (2018). doi:10.1016/j.imavis.2018.09.017

13. Stork, D.G.: Character and document research in the open mind initiative. In: Proceedings of the Fifth International Conference on Document Analysis and Recognition. ICDAR '99 (Cat. No.PR00318), pp. 1-12 (1999). doi:10.1109/ICDAR.1999.791712

14. Ahn, L.v., Dabbish, L.: Labeling images with a computer game, 319-326 (2004). doi:10.1145/985692.985733

15. Russell, B.C., Torralba, A., Murphy, K.P., Freeman, W.T.: Labelme: A database and web-based tool for image annotation. International Journal of Computer Vision 77 (2008). doi:10.1007/s11263-007-0090-8

16. Sekachev, B., Manovich, N., Zhavoronkov, A.: Computer Vision Annotation Tool: A Universal Approach to Data Annotation. https://software.intel.com/content/www/us/en/develop/articles/ computer-vision-annotation-tool-a-universal-approach-to-data-annotation.html Accessed 2021-06-01

17. Tzutalin: Labellmg. https://github.com/tzutalin/labelImg Accessed 2021-06-01

18. Wada, K.: labelme: Image Polygonal Annotation with Python. https://github.com/wkentaro/labelme (2016)

19. Microsoft: Visual Object Tagging Tool. https://github.com/microsoft/VoTT Accessed 2021-07-01

20. Dutta, A., Zisserman, A.: The VIA annotation software for images, audio and video. In: Proceedings of the 27th ACM International Conference on Multimedia. MM '19. ACM, New York, NY, USA (2019) doi:10.1145/3343031.3350535. https://doi.org/10.1145/3343031.3350535

21. Dutta, A., Gupta, A., Zissermann, A.: VGG Image Annotator (VIA) (2016). http://www.robots.ox.ac.uk/texttildelowvgg/software/via/ Accessed 2021-06-09

22. Rajkomar, A., Dean, J., Kohane, I.: Machine learning in medicine. New England Journal of Medicine 380(14), 1347-1358 (2019)

23. Sidey-Gibbons, J.A., Sidey-Gibbons, C.J.: Machine learning in medicine: a practical introduction. BMC medical research methodology 19(1), 1-18 (2019)

24. Wang, F., Casalino, L.P., Khullar, D.: Deep learning in medicine-promise, progress, and challenges. JAMA internal medicine 179(3), 293-294 (2019)

25. Yushkevich, P.A., Gao, Y., Gerig, G.: Itk-snap: An interactive tool for semi-automatic segmentation of multi-modality biomedical images. In: 2016 38th Annual International Conference of the IEEE Engineering in Medicine and Biology Society (EMBC), pp. 3342-3345 (2016). doi:10.1109/EMBC.2016.7591443

26. Rubin-Lab: ePAD: web-based platform for quantitative imaging in the clinical workflow. [Online; Stand 13.05.2021] (2014). https://epad.stanford.edu/

27. Gupta, G., Gupta, A.: TrainingData.io. [Online; Stand 13.05.2021] (2019). https://docs.trainingdata.io/

28. Gupta, G.: TrainingData.io: Al Assisted Image \& Video Training Data Labeling Scale. [Online; Stand 13.05.2021] (2019). https://github.com/trainingdata/AIAssistedImageVideoLabelling/

29. Philbrick, K., Weston, A., Akkus, Z., Kline, T., Korfiatis, P., Sakinis, T., Kostandy, P., Boonrod, A., Zeinoddini, A., Takahashi, N., Erickson, B.: Ril-contour: a medical imaging dataset annotation tool for and with deep learning. Journal of Digital Imaging 32 (2019). doi:10.1007/s10278-019-00232-0

30. Leibetseder, A., Münzer, B., Schoeffmann, K., Keckstein, J.: Endometriosis annotation in endoscopic videos. In 2017 IEEE International Symposium on Multimedia (ISM), pp. 364-365 (2017). doi:10.1109/ISM.2017.69

31. Guo, Y.B., Matuszewski, B.J.: Giana polyp segmentation with fully convolutional dilation neural networks. In: VISIGRAPP, pp. 632-641 (2019)

32. Mahony, N.O., Campbell, S., Carvalho, A., Harapanahalli, S., Velasco-Hernandez, G., Krpalkova, L., Riordan, D., Walsh, J.: Deep learning vs. traditional computer vision. doi:10.1007/978-3-030-17795-9. 1910.13796

33. Silva, J., Histace, A., Romain, O., Dray, X., Granado, B.: Toward embedded detection of polyps in wce images for early diagnosis of colorectal cancer. International journal of computer assisted radiology and surgery $\mathbf{9}$ 283-293 (2014). doi:10.1007/s11548-013-0926-3

34. Qadir, H.A., Balasingham, I., Solhusvik, J., Bergsland, J., Aabakken, L., Shin, Y.: Improving automatic polyp detection using $\mathrm{cnn}$ by exploiting temporal dependency in colonoscopy video. IEEE journal of biomedical and health informatics 24(1), 180-193 (2019)

35. Hasan, M.M., Islam, N., Rahman, M.M.: Gastrointestinal polyp detection through a fusion of contourlet transform and neural features. Journal of King Saud University-Computer and Information Sciences (2020)

36. Sun, X., Wang, D., Zhang, C., Zhang, P., Xiong, Z., Cao, Y., Liu, B., Liu, X., Chen, S.: Colorectal polyp detection in real-world scenario: Design and experiment study. In: 2020 IEEE 32nd International Conference on Tools with Artificial Intelligence (ICTAI), pp. 706-713 (2020). IEEE

37. Lambert, R.f.: Endoscopic classification review group. update on the paris classification of superficial neoplastic lesions in the digestive tract. Endoscopy 37(6), 570-578 (2005)

38. Zhang, X., Chen, F., Yu, T., An, J., Huang, Z., Liu, J., Hu, W., Wang, L., Duan, H., Si, J.: Real-time gastric polyp detection using convolutional neural networks. PloS one 14(3), 0214133 (2019)

39. Jha, D., Ali, S., Tomar, N.K., Johansen, H.D., Johansen, D., Rittscher, J., Riegler, M.A., Halvorsen, P.: Real-time polyp detection, localization and segmentation in colonoscopy using deep learning. leee Access $\mathbf{9}$, 40496-40510 (2021)

40. Bernal, J., Tajkbaksh, N., Sánchez, F.J., Matuszewski, B.J., Chen, H., Yu, L., Angermann, Q., Romain, O., Rustad, B., Balasingham, I., et al.: Comparative validation of polyp detection methods in video colonoscopy: results from the miccai 2015 endoscopic vision challenge. IEEE transactions on medical imaging 36(6), 1231-1249 (2017)

41. Shackleton, V.: Boredom and repetitive work: a review. Personnel Review (1981)

42. Pal, S.K., Pramanik, A., Maiti, J., Mitra, P.: Deep learning in multi-object detection and tracking: state of the 
art. Applied Intelligence, 1-30 (2021)

43. Li, Y., Zhang, X., Li, H., Zhou, Q., Cao, X., Xiao, Z.: Object detection and tracking under complex environment using deep learning-based Ipm. IET computer vision 13(2), 157-164 (2019)

44. Bradski, G.: The OpenCV Library. Dr. Dobb's Journal of Software Tools (2000)

45. Grabner, H., Grabner, M., Bischof, H.: Real-time tracking via on-line boosting. In: BMVC (2006)

46. Babenko, B., Yang, M., Belongie, S.: Visual tracking with online multiple instance learning. In: 2009 IEEE Conference on Computer Vision and Pattern Recognition, pp. 983-990 (2009)

47. Henriques, J., Caseiro, R., Martins, P., Batista, J.: Exploiting the circulant structure of tracking-by-detection with kernels, vol. 7575, pp. 702-715 (2012)

48. Lukezic, A., Vojir, T., Cehovin Zajc, L., Matas, J., Kristan, M.: Discriminative correlation filter with channel and spatial reliability. In: Proceedings of the IEEE Conference on Computer Vision and Pattern Recognition (CVPR) (2017)

49. Gong, F., Yue, H., Yuan, X., Gong, W., Song, T.: Discriminative Correlation Filter for Long-Time Tracking. The Computer Journal 63(3), 460-468 (2019). doi:10.1093/comjnl/bxz049. https://academic.oup.com/comjnl/article-pdf/63/3/460/33106425/bxz049.pdf

50. Kalal, Z., Mikolajczyk, K., Matas, J.: Forward-backward error: Automatic detection of tracking failures. In: 2010 20th International Conference on Pattern Recognition, pp. 2756-2759 (2010). doi:10.1109/ICPR.2010.675

51. OpenCV: MedianFlow Tracker Class Reference. https://docs.opencv.org/4.3.0/d7/d86/classcv \_1\_1TrackerMedianFlow.html\\#details Accessed 2021-05-12

52. Draper, B.A., Bolme, D.S., Beveridge, J., Lui, Y.: Visual object tracking using adaptive correlation filters. In: 2010 IEEE Conference on Computer Vision and Pattern Recognition (CVPR), pp. 2544-2550. IEEE Computer Society, Los Alamitos, CA, USA (2010). doi:10.1109/CVPR.2010.5539960. https://doi.ieeecomputersociety.org/10.1109/CVPR.2010.5539960

53. Kalal, Z., Mikolajczyk, K., Matas, J.: Tracking-learning-detection. IEEE Trans. Pattern Anal. Mach. Intell. 34(7), 1409-1422 (2012). doi:10.1109/TPAMI.2011.239 